\title{
UNSTEADY FLUID MOTION BETWEEN TWO INFINITE WALLS UNDER VARIABLE BODY FORCE
}

\author{
R. A. Lemdiasov", \\ Department of Electrical and Computer Engineering, Worcester Polytechnic Institute, Worcester MA \\ V. M. Tenishev ${ }^{\dagger}$, \\ Department of Atmospheric, Oceanic and Space Sciences, University of Michigan, Ann Arbor MI \\ A. I. Fedoseyev \\ Center for Microgravity and Materials Research University of Alabama in Huntsville, Huntsville AL, and CFD \\ Research Corporation, Huntsville AL
}

\begin{abstract}
$\underline{\text { Abstract }}$
We considered an incompressible fluid motion driven by space-dependent body force. For a one-dimensional case, the problem was solved analytically, with the arbitrary choice of body force coordinate dependence. It was shown that unsteady fluid flow can be represented as a series of separate modes, each with its own characteristic response time. The analytical solution was proved using a corresponding numerical simulation.
\end{abstract}

\section{Introduction}

The problem of fluid motion induced by a variable body force has many applications. There are several ways to apply this force to the fluid. One is the nonuniform distribution of particles settling in the container. These particles interact with nearby fluid, initiating its motion. On the other hand, moving fluid exerts a force on the particles and influences their motion.

The literature on the dynamics of suspensions is very extensive and due to the limited scope of this study, it is impossible to cover the topic completely. We reviewed a sampling of studies closely related to the subject of this paper.

There are many studies of sedimentation of spheres in a container, which assume that particles are distributed uniformly in the area no closer than the radius of a particle to the container wall. Close to the wall (closer than $a$ ), the concentration of particles is zero, because no particle can be closer to the wall, than its own radius.

\footnotetext{
*, Graduate Research Assistant

${ }^{\dagger}$ Graduate Research Assistant

\$ Professor
}

Bruneau et al. ${ }^{1}$ stated this problem in a simple way: there are two infinitely high walls with particles distributed between them (no closer than $a$ ). They discussed only a steady state problem, with noslip boundary conditions, and introduced a zero netflux condition (no net fluid flux through the cross section of the container):

$$
\int_{\text {cross section }} w d S=0
$$

One reason to introduce this condition is that the purpose of the analytical solution is to investigate fluid motion in a finite real container, where the zero net-flux condition is obviously satisfied. Introducing this condition makes it possible to solve a system of hydrodynamic equations. The authors managed to solve the problem exactly, but assuming (a) the existence of the boundary layer, with constant stress inside, and (b) Poiseuille flow in the inner regions of the container. They obtained the expressions for a fluid velocities on the edge of the wall layer and in the center of the container. These velocities are found to be of the order of $V_{0} s$, where $V_{0}$ is the Stokes 
velocity of settling particles, and $s$ is a volume fraction of solid particles.

In another work made by Bruneau et $\mathrm{al}^{2}$, they discussed the same steady state problem in the geometry of an infinitely high rectangular container. As before, they looked for solution as a superposition of constant stress flow and Poiseuille flow. An expression was obtained for fluid velocity inside the container. It was shown that if the length of one side of the container tends towards infinity, then the solution approaches the one for one-dimensional case. The dependence of an intrinsic convection on a suspension concentration was estimated. An accurate description of the intrinsic convection, at moderate and high concentrations, would require an analysis of multi-particle hydrodynamic interactions in the presence of walls.

Beenakker and Mazur ${ }^{3}$ studied a possible dependence of sedimentation on container-shape. The phenomenon of intrinsic convection was predicted: an intrinsic, microscopic density inhomogeneity at the container wall causes a macroscopic vortex motion of the mean volume velocity.

A phenomenological theory of this effect, in terms of two coupled velocity fields and an effective boundary condition, was developed by Nozières ${ }^{4}$.

The above authors agreed on the following: in order for fluid bulk motion in the container to exist, particles should not be distributed uniformly throughout the container. The difference of particle concentration in different parts of the container results in pressure gradient in the fluid, causing fluid motion.

In this work, we focus on the unsteady large-scale fluid dynamics, and discuss arbitrary spatial distribution of the body force throughout the container. As an example, we introduced Gaussian distribution of body force around the center of the container.

\section{Physical Formulation of the Problem and} Governing Equations

We considered a fluid flow between two infinitely high walls separated by a distance $L_{0}$ (Figure 1 ).

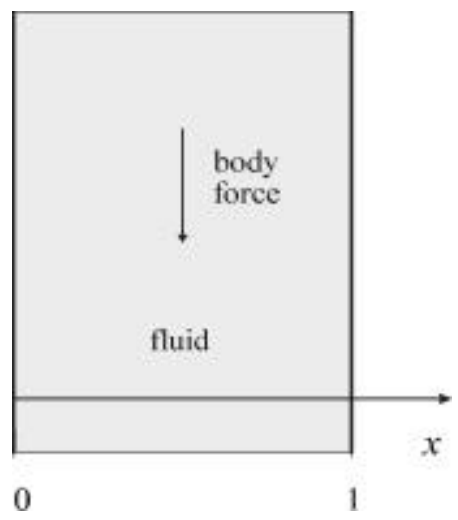

Figure 1: Geometry of the problem.

The fluid experiences body force $\mathbf{f}$, which depends on coordinate $x$ and acts in the $z$-direction. We do not specify for now the origin of this body force, which acts on the fluid and arouses a motion. We assume the fluid to be Newtonian and use the following governing equations.

Conservation of mass:

$$
\nabla \cdot \mathbf{U}=0 \text {. }
$$

Conservation of momentum:

$$
\rho_{f}\left(\frac{\partial \mathbf{U}}{\partial t}+(\mathbf{U} \cdot \nabla) \mathbf{U}\right)=-\nabla p+\nabla \cdot \tau+\mathbf{f},
$$

where $\rho_{f}$ is fluid density, $\mathbf{U}$ is fluid velocity, $\mathbf{f}$ is a body force, and $\tau$ is the viscous stress tensor for a Newtonian fluid defined as:

$$
\tau=\mu\left(\nabla \mathbf{U}+\nabla \mathbf{U}^{T}\right)
$$

Initially, fluid is assumed to be stationary. Below, we investigate Gaussian distribution of body force around the center of the container $x_{0}=L_{0} / 2$. Therefore, distribution function is seen as:

$$
G(x)=\frac{A}{\left(2 \pi \Delta^{2}\right)^{1 / 2}} \exp \left(-\frac{\left(x-x_{0}\right)^{2}}{2 \Delta^{2}}\right),
$$

where the parameters are $x_{0}=\frac{1}{2} L_{0}, \Delta=\frac{1}{5} L_{0}$ (the coefficient $A=1.013$ is introduced in order to ensure that $\left.\int_{0}^{L_{0}} G(x) d x=1\right)$. The vector of body force is $\mathbf{f}=\left(\begin{array}{lll}0 & 0 & -f L_{0} G(x)\end{array}\right)$, where $f$ is the magnitude of averaged body force. It is obvious that fluid motion will occur only in the $z$ direction. In onedimensional case Equation (2) can be rewritten as:

$$
\frac{\partial p}{\partial x}=0,
$$


with initial condition:

$$
\rho_{f} \frac{\partial w}{\partial t}=-\frac{\partial p}{\partial z}+\mu \frac{\partial^{2} w}{\partial x^{2}}-f L_{0} G(x),(6)
$$

$$
w(x, 0)=0,
$$

and with boundary conditions:

$$
w(0, t)=w\left(L_{0}, t\right)=0 .
$$

Furthermore, we introduce one more condition by requiring a zero fluid flux through a cross-section of the container:

$$
\int_{0}^{L_{0}} w(x, t) d x=0 .
$$

Later, we solve this PDE analytically.

The governing equations can be nondimensionalized with respect to the following quantities:

$$
w_{0}=f L_{0}^{2} / \mu, \quad \tau=\rho_{f} L_{0}^{2} / \mu, \quad \Delta_{0}=L_{0} .
$$

It is easy to see that pressure $p$ is a linear function of the $z$ coordinate. We can, therefore, introduce a nondimensional function $\pi(t)$ as follows:

$$
p=f \pi(t) z
$$

The non-dimensional formulation is:

$$
\frac{\partial \tilde{w}}{\partial \tilde{t}}=-\pi(\tilde{t})+\frac{\partial^{2} \tilde{w}}{\partial \tilde{x}^{2}}-\tilde{G}(\tilde{x})
$$

with initial condition:

$$
\tilde{w}(\tilde{x}, 0)=0,
$$

and with boundary conditions:

$$
\tilde{w}(0, \tilde{t})=\tilde{w}(1, \tilde{t})=0,
$$

and a zero fluid flux condition:

$$
\int_{0}^{1} \tilde{w}(\tilde{x}, \tilde{t}) d \tilde{x}=0 .
$$

Here, the distribution function is:

$$
\tilde{G}(\tilde{x})=\frac{A}{\left(2 \pi \tilde{\Delta}^{2}\right)^{1 / 2}} \exp \left(-\frac{\left(\tilde{x}-\tilde{x}_{0}\right)^{2}}{2 \tilde{\Delta}^{2}}\right),
$$

Later, we omitted the tilde sign for non-dimensional parameters.

\section{Analytical Solution of the Steady State and}

\section{Unsteady Problems}

\section{A. Steady state case}

We write the governing equation as:

$$
\frac{\partial^{2} w_{s t}}{\partial x^{2}}=\pi_{s t}+G(x)
$$

with boundary conditions:

$$
w_{s t}(0)=0, \quad w_{s t}(1)=0,
$$

$$
\int_{0}^{1} w_{s t}(x) d x=0 .
$$

We seek a solution as:

$$
w_{s t}(x)=\sum_{k=1}^{\infty} a_{k} \sin (\pi k x) .
$$

Substitution of (20) into (17) will result in:

$$
-a_{k}(\pi k)^{2}=\pi_{s t, k}+G_{k}, \quad k=1, \ldots, \infty,
$$

where

$$
\begin{aligned}
\pi_{s t, k}=\pi_{s t} 2 \int_{0}^{1} \sin (\pi k x) d x & =\pi_{s t} \frac{2}{\pi k}\left(1-(-1)^{k}\right) \\
k & =1, \ldots, \infty
\end{aligned}
$$

and

$$
G_{k}=2 \int_{0}^{1} G(x) \sin (\pi k x) d x, \quad k=1, \ldots, \infty .
$$

We use a zero net-flux condition (19):

$$
\begin{aligned}
& 0=\int_{0}^{1} w_{s t}(x) d x=\sum_{k=1}^{\infty} a_{k} \frac{\left(1-(-1)^{k}\right)}{\pi k}= \\
& =-\sum_{k=1}^{\infty} \frac{\left(1-(-1)^{k}\right)}{(\pi k)^{3}}\left[\frac{2 \pi}{\pi k}\left(1-(-1)^{k}\right)+G_{k}\right] .
\end{aligned}
$$

From this one can obtain $\pi_{s t}$ :

$$
\pi_{s t}=-\sum_{k=1}^{\infty} \frac{G_{k}\left(1-(-1)^{k}\right)}{(\pi k)^{3}} / \sum_{k=1}^{\infty} \frac{2\left(1-(-1)^{k}\right)^{2}}{(\pi k)^{4}}
$$

And, finally, the steady state solution is:

$w_{s t}(x)=-\sum_{k=1}^{\infty} \frac{2 \pi_{s t}\left(1-(-1)^{k}\right) \frac{1}{\pi k}+G_{k}}{(\pi k)^{2}} \sin (\pi k x)$,

\section{B. Unsteady case}

We introduce new variables:

$$
\begin{aligned}
& \pi(t)=\pi_{s t}+\pi^{*}(t), \\
& w=w_{s t}+w^{*},
\end{aligned}
$$

obtaining:

$$
\frac{\partial w^{*}}{\partial t}=-\pi^{*}(t)+\frac{\partial^{2} w^{*}}{\partial x^{2}},
$$

with initial condition:

$$
w^{*}(x, 0)=-w_{s t}(x),
$$

where $w_{s t}(x)$ is a steady state solution found in (25). We also have the following conditions:

$$
w^{*}(0, t)=0, \quad w^{*}(1, t)=0,
$$




$$
\int_{0}^{1} w^{*}(x, t) d x=0 .
$$

The solution of (28) has the general form of:

$$
\begin{aligned}
& w^{*}=\sum_{n=1}^{\infty} \exp \left(-\lambda_{n}^{2} t\right)\left(A_{n} \cos \left(\lambda_{n} x\right)+B_{n} \sin \left(\lambda_{n} x\right)\right)+ \\
& +\int_{t}^{\infty} \pi^{*}\left(t^{\prime}\right) d t^{\prime},
\end{aligned}
$$

where eigenvalues $\lambda_{n}$ are still unknown. Applying zero net flux condition, we get:

$$
\begin{aligned}
& 0=\sum_{n=1}^{\infty} \exp \left(-\lambda_{n}^{2} t\right)\left(A_{n} \frac{\sin \left(\lambda_{n}\right)}{\lambda_{n}}+B_{n} \frac{1-\cos \left(\lambda_{n}\right)}{\lambda_{n}}\right)+ \\
& +\int_{t}^{\infty} \pi^{*}\left(t^{\prime}\right) d t^{\prime} .
\end{aligned}
$$

From here we have an expression for $w^{*}$ as:

$$
\begin{aligned}
w^{*}(x, t)=\sum_{n=1}^{\infty} \exp \left(-\lambda_{n}^{2} t\right)\left(A_{n}\left(\cos \left(\lambda_{n} x\right)-\frac{\sin \left(\lambda_{n}\right)}{\lambda_{n}}\right)+\right. \\
\left.+B_{n}\left(\sin \left(\lambda_{n} x\right)-\frac{1-\cos \left(\lambda_{n}\right)}{\lambda_{n}}\right)\right) .
\end{aligned}
$$

Applying boundary conditions, we come to an expression for $\lambda_{n}$ :

$$
\lambda_{n} \sin \left(\lambda_{n}\right)=2\left(1-\cos \left(\lambda_{n}\right)\right) .
$$

This equation gives us two sets of solutions:

$$
\sin (\lambda / 2)=0, \quad\left(\lambda_{k}=2 \pi k, k=1, \ldots, \infty\right),(36)
$$

and

$$
\tan (\lambda / 2)=\lambda / 2
$$

The first ten solutions $\lambda_{n}$ of Equation (35) are shown in a Table 1 . It can be shown, that if $\lambda_{n}$ satisfies (37), then it also satisfies the following relations:

$$
\cos \left(\lambda_{n}\right)=\frac{1-\left(\lambda_{n} / 2\right)^{2}}{1+\left(\lambda_{n} / 2\right)^{2}}, \sin \left(\lambda_{n}\right)=\frac{\lambda_{n}}{1+\left(\lambda_{n} / 2\right)^{2}}
$$

Eigenvalues for velocity $w^{*}$ are given by the expression $-\lambda_{n}^{2}$, shown in Table 2. Finally, we obtain eigenfunctions $f_{n}(x)$ :

$f_{n}(x)=\left\{\begin{array}{l}\sin \left(\lambda_{n} x\right), \text { if } \lambda_{n} \text { satisfies (36) } \\ \frac{2}{\lambda_{n}}\left(\cos \left(\lambda_{n} x\right)-1\right)+\sin \left(\lambda_{n} x\right), \text { if } \lambda_{n} \text { satisfies (37) }\end{array}\right.$

Functions $f_{n}(x)$ are found to be mutually orthogonal:

$$
\int_{0}^{1} f_{n}(x) f_{m}(x) d x=0, \quad \text { if } n \neq m .
$$

They also satisfy the following relations:

$$
\int_{0}^{1} f_{n}^{2}(x) d x=\frac{1}{2}, n=1, \ldots, \infty .
$$

Solution to the unsteady problem (28) - (31) can be expressed as:

$$
w^{*}=\sum_{n=1}^{\infty} C_{n} \exp \left(-\lambda_{n}^{2} t\right) f_{n}(x),
$$

where coefficients $C_{n}$ are given by:

$$
C_{n}=-2 \int_{0}^{1} w_{s t}(x) f_{n}(x) d x .
$$

We can also introduce non-dimensional response time parameters $\tau_{\text {res, } n}$ :

$$
\tau_{\text {res, } n}=1 / \lambda_{n}^{2} \text {. }
$$

This represents the time scale for the corresponding modes $f_{n}(x)$ exponential decay. Finally, the analytical solution of unsteady problem (28) - (31) is given as:

$$
w(x, t)=w_{s t}(x)+\sum_{n=1}^{\infty} C_{n} \exp \left(-\lambda_{n}^{2} t\right) f_{n}(x) .
$$

\section{Galerkin FEM Formulation and solution of the Problem}

According to the Finite Element Method, velocity distribution between the walls is represented by the following expression:

$$
w(x)=\sum_{n=1}^{N} w_{n} \varphi_{n}(x),
$$

where $\varphi_{n}(x)$ are the basic functions. $\varphi_{n}(x)$ is chosen to be equal to unity in $n$-th node and equal to zero at all other nodes. Nodes are chosen to be equidistant. Using this approximation, from (12) we obtain the following linear system for node velocities $w_{n}$ :

$$
\mathbf{M} \frac{d \vec{w}}{d t}=-\pi(t) \vec{b}-\mathbf{N} \vec{w}-\vec{G}
$$

Here we used the following notations:

$$
\begin{array}{rlrl}
\mathbf{M}_{j i} & =\int_{0}^{1} \varphi_{j} \varphi_{i} d x, \mathbf{N}_{j i}=\int_{0}^{1} \frac{d \varphi_{j}}{d x} \frac{d \varphi_{i}}{d x} d x \\
b_{i} & =\int_{0}^{1} \varphi_{i} d x, & G_{i} & =\int_{0}^{1} \varphi_{i} G(x) d x
\end{array}
$$

Zero net flux condition implies:

$$
\sum_{n=1}^{N} w_{n}=0,
$$

or, in other words: 


$$
\overrightarrow{1}^{T} \vec{w}=0,
$$

where $\overrightarrow{1}$ is a vector with all components equal to unity. In the steady state case we have:

$$
-\pi_{s t} \vec{b}-\mathbf{N} \vec{w}_{s t}-\vec{G}=0 .
$$

Multiplying (51) by $\overrightarrow{1}^{T} \mathbf{N}^{-1}$, we obtain:

$$
-\overrightarrow{1}^{T} \mathbf{N}^{-1} \vec{b} \pi_{s t}(t)-\overrightarrow{1}^{T} \mathbf{N}^{-1} \vec{G}=0 .
$$

From here:

$$
-\pi_{s t}=\frac{\left(\overrightarrow{1}^{T} \mathbf{N}^{-1} \vec{G}\right)}{\left(\overrightarrow{1}^{T} \mathbf{N}^{-1} \vec{b}\right)}
$$

and

$$
\vec{w}_{s t}=\mathbf{N}^{-1}\left(\vec{b} \frac{\left(\overrightarrow{1}^{T} \mathbf{N}^{-1} \vec{G}\right)}{\left(\overrightarrow{1}^{T} \mathbf{N}^{-1} \vec{b}\right)}-\vec{G}\right) .
$$

Expression (54) represents a steady state solution to our problem. Now we derive ODE for velocity of the fluid $\vec{w}$. Multiplying (51) by $\overrightarrow{1}^{T} \mathbf{M}^{-1}$, we obtain:

$$
-\pi(t)=\frac{\overrightarrow{1}^{T} \mathbf{M}^{-1} \mathbf{N} \vec{w}+\left(\overrightarrow{1}^{T} \mathbf{M}^{-1} \vec{G}\right)}{\left(\overrightarrow{1}^{T} \mathbf{M}^{-1} \vec{b}\right)} .
$$$$
0=-\pi(t) \overrightarrow{1}^{T} \mathbf{M}^{-1} \vec{b}-\overrightarrow{1}^{T} \mathbf{M}^{-1} \mathbf{N} \vec{w}-\overrightarrow{1}^{T} \mathbf{M}^{-1} \vec{G}
$$

Substituting this expression for $\rho_{f} \frac{\partial w}{\partial t}=-\frac{\partial p^{\prime}}{\partial z}+\mu \frac{1}{r} \frac{\partial}{\partial r}\left(r \frac{\partial w}{\partial r}\right)-\rho_{f} g_{0}(\delta-1) s \quad$ into (47) we obtain:

$$
\frac{d \vec{w}}{d t}=\mathbf{M}^{-1} \vec{b} \frac{\overrightarrow{1}^{T} \mathbf{M}^{-1} \mathbf{N} \vec{w}+\left(\overrightarrow{1}^{T} \mathbf{M}^{-1} \vec{G}\right)}{\left(\overrightarrow{1}^{T} \mathbf{M}^{-1} \vec{b}\right)}-\mathbf{M}^{-1} \mathbf{N} \vec{w}-\mathbf{M}^{-1} \vec{G}
$$

From here:

$$
\begin{aligned}
\frac{d \vec{w}}{d t} & =\left(\frac{\mathbf{M}^{-1} \vec{b}\left(\overrightarrow{1}^{T} \mathbf{M}^{-1} \mathbf{N}\right)}{\left(\overrightarrow{1}^{T} \mathbf{M}^{-1} \vec{b}\right)}-\mathbf{M}^{-1} \mathbf{N}\right) \vec{w}+ \\
& +\left(\frac{\mathbf{M}^{-1} \vec{b}\left(\overrightarrow{1}^{T} \mathbf{M}^{-1} \vec{G}\right)}{\left(\overrightarrow{1}^{T} \mathbf{M}^{-1} \vec{b}\right)}-\mathbf{M}^{-1} \vec{G}\right) .
\end{aligned}
$$

Let us denote:

$$
\begin{gathered}
\mathbf{A}=\left(\frac{\mathbf{M}^{-1} \vec{b}\left(\overrightarrow{1}^{T} \mathbf{M}^{-1} \mathbf{N}\right)}{\left(\overrightarrow{1}^{T} \mathbf{M}^{-1} \vec{b}\right)}-\mathbf{M}^{-1} \mathbf{N}\right) \\
\vec{B}=\frac{\mathbf{M}^{-1} \vec{b}\left(\overrightarrow{1}^{T} \mathbf{M}^{-1} \vec{G}\right)}{\left(\overrightarrow{1}^{T} \mathbf{M}^{-1} \vec{b}\right)}-\mathbf{M}^{-1} \vec{G} .
\end{gathered}
$$

and

Then

$$
\frac{d \vec{w}}{d t}=\mathbf{A} \vec{w}+\vec{B}
$$

with zero initial condition:

$$
\vec{w}_{0}=0 .
$$

This system can be simulated using numerical methods. To solve this problem numerically, we used 200 and more inner nodes between the walls. Unsteady fluid motion can be described in two ways: analytically with (45) and numerically with (60) (61). Results obtained using both methods proved to be in a very good agreement. In Figure 2, we plotted flow profiles at several consecutive time moments. We also calculated the eigenvalues $\mu_{n}$ of matrix $\mathbf{A}$ and compared them to those found analytically. Table 3 shows the eigenvalues of matrix $\mathbf{A}$. Comparison of these eigenvalues with those in Table 2 shows a discrepancy in the first ten eigenvalues of no more than $0.25 \%$. This discrepancy increases with the eigenvalue number and is explained by a numerical error.

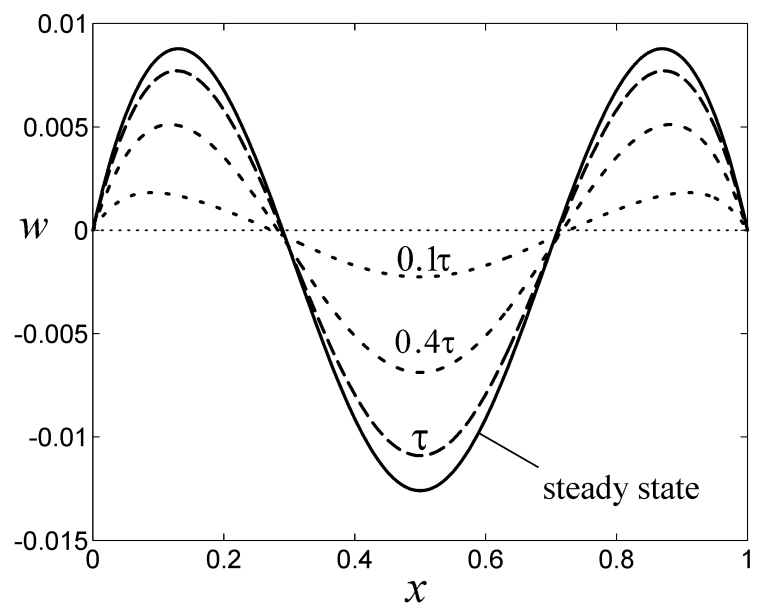

Figure 2: Development of fluid flow between infinite walls at several consecutive time moments.
Tables

\begin{tabular}{|c|c|}
\hline$\lambda_{1}=6.2832$ & $\lambda_{6}=21.8083$ \\
\hline$\lambda_{2}=8.9868$ & $\lambda_{7}=25.1327$ \\
\hline$\lambda_{3}=12.5664$ & $\lambda_{8}=28.1324$ \\
\hline$\lambda_{4}=15.4505$ & $\lambda_{9}=31.4159$ \\
\hline$\lambda_{5}=18.8496$ & $\lambda_{10}=34.4415$ \\
\hline
\end{tabular}

Table 1. First ten eigenvalues found from Eq. (35). 


\begin{tabular}{|l|l|}
\hline$-\lambda_{1}^{2}=-39.4786$ & $-\lambda_{6}^{2}=-475.602$ \\
\hline$-\lambda_{2}^{2}=-80.7626$ & $-\lambda_{7}^{2}=-631.653$ \\
\hline$-\lambda_{3}^{2}=-157.914$ & $-\lambda_{8}^{2}=-791.432$ \\
\hline$-\lambda_{4}^{2}=-238.718$ & $-\lambda_{9}^{2}=-986.959$ \\
\hline$-\lambda_{5}^{2}=-355.307$ & $-\lambda_{10}^{2}=-1186.217$ \\
\hline
\end{tabular}

Table 2. Eigenvalues for velocity, obtained analytically.

\begin{tabular}{|c|c|}
\hline$\mu_{1}=-39.4816$ & $\mu_{6}=-476.069$ \\
\hline$\mu_{2}=-80.7776$ & $\mu_{7}=-632.470$ \\
\hline$\mu_{3}=-157.9646$ & $\mu_{8}=-792.724$ \\
\hline$\mu_{4}=-238.8384$ & $\mu_{9}=-988.951$ \\
\hline$\mu_{5}=-355.5637$ & $\mu_{10}=-1189.114$ \\
\hline
\end{tabular}

Table 3. Eigenvalues for velocity, obtained numerically.

\section{Conclusions}

We investigated the fluid motion driven by $x$ coordinate dependent body force in a onedimensional case. Analytically, solution of the problem, the flow field, was obtained for unsteady and steady state cases. As for time dependence of fluid flow, fluid velocity was found to be the superposition of specific modes, each exponentially decaying with its own characteristic time. These times were found by solving a transcendental equation.

The Finite Element Method was used for verification of analytic results. The numerical results for eigenvalues are in good agreement the ones found analytically. The discrepancy was decreasing with refining the accuracy of numerical method by using more detailed meshes. Numerical investigations for flow field were performed and numerical results agreed well the analytical ones found earlier.

\section{Acknowledgments}

Authors are grateful to Profs. A.N. Alexandrou and N.A. Gatsonis for guidance in preparing this paper. Authors would also like to thank Ms. Lynne Carver for her invaluable help.

\section{References}

${ }^{1}$ D. Bruneau, F. Feuillebois, R. Anthore, and E. J. Hinch, "Intrinsic convection in a settling suspension," Phys. Fluids 8, 2236 (1996)

${ }^{2}$ D. Bruneau, F. Feuillebois, J. Blawzdziewicz, and R. Anthore, "Three-dimensional intrinsic convection in dilute and dense dispersions of settling spheres," Phys. Fluids 10, 55 (1998)

${ }^{3}$ C. W. J. Beenakker and P. Mazur, "Is sedimentation container-shape dependent," Phys. Fluids 28, 3203 (1985)

4 P. Nozières, "A local coupling between sedimentation and convection: Application to Beenakker-Mazur effect," Physica A 147, 219 (1987)

${ }^{5}$ O. C. Zienkiewic, "The finite element method in structural and continuum mechanics: numerical solution of problems in structural and continuum mechanics", London, New York, etc. McGrawHill (1967)

${ }^{6}$ B. U. Felderhof, "Sedimentation and convective flow in suspensions of spherical particles," Physica A 153, 217 (1988)

7 U. Geigenmüller and P. Mazur, "Intrinsic convection near a meniscus," Physica A 171, 475 (1991)

${ }^{8}$ U. Geigenmüller and P. Mazur, "Sedimentation of homogeneous suspensions in finite vessels," Journal of Statistical Physics 53, 137 (1988)

${ }^{9}$ Y. Peysson and E. Guazzelli, "An experimental investigation of intrinsic convection in sedimenting suspension," Phys. Fluids 10 (1), 44 (1998)

10 G. K. Batchelor, "Sedimentation of a dilute dispersion of spheres," J. Fluid Mech. 52, 245 (1972)

${ }^{11}$ J. Blawzdziewicz and F. Feuillebois, "Calculation of an effective slip in a settling suspension at a vertical wall," Fluid Mech. Res. 22, 81 (1996) 